\title{
On the role of $\mathrm{Sm}$ in solidification of Al-Sm metallic glasses
}

\author{
G.B. Bokas ${ }^{\mathrm{a}}$, L. Zhao ${ }^{\mathrm{a}}$, J.H. Perepezko ${ }^{\mathrm{a}}$, I. Szlufarska ${ }^{\mathrm{a}, *}$ \\ ${ }^{a}$ University of Wisconsin-Madison, Department of Materials Science and Engineering,1509 \\ University Ave, Madison 53706, USA
}

\begin{abstract}
During the solidification of Al-Sm metallic glasses the evolution of the supercooled liquid atomic structure has been identified with an increasing population of icosahedral-like clusters with increasing Sm concentration. These clusters exhibit slower kinetics compared to the remaining clusters in the liquid leading to enhanced amorphous phase stability and glass forming ability (GFA). Maximum icosahedral-ordering and atomic packing density have been found for the $\mathrm{Al}_{90} \mathrm{Sm}_{10}$ and $\mathrm{Al}_{85} \mathrm{Sm}_{15}$ alloys, respectively, whereas minimum cohesive energy has been found for the $\mathrm{Al}_{93} \mathrm{Sm}_{7}$ which is consistent with the range of compositions (from $\mathrm{Al}_{92} \mathrm{Sm}_{8}$ to $\mathrm{Al}_{84} \mathrm{Sm}_{16}$ ) found experimentally with high GFA.
\end{abstract}

Keywords: metallic glass; short-range order; molecular dynamics; solidification.

Metallic glasses (MGs) are known to have attractive mechanical properties such as high strength and high elastic limit, good corrosion and wear resistance and good biocompatibility [1, 2, 3]. However, the low glass forming ability (GFA) of some MGs has so far hindered synthesis of large MG specimens such 5 as Al-base systems and therefore limited engineering applications of these materials. Many efforts have been devoted towards developing alloys with high GFA [1, 4, 5]. In particular, a number of studies focused on understanding of the MGs atomic structure and its relation to the MGs' mechanical properties

\footnotetext{
*Author to whom correspondence should be addressed

Email address: szlufarska@wisc.edu (I. Szlufarska)
}

Preprint submitted to Scripta Materialia

June 27, 2016 
and GFA [6, 7, 8]. It is well known that the MGs lack long range order but exhibit short- and medium-range orders [8]. Commonly, short-range order (SRO) is characterized by Voronoi polyhedrons (VPs) and medium-range order (MRO) by the arrangement of VPs into networks [9, 10, 11, 12, 13.

Among Al-based rare earth (Al-RE) metal alloys ( $\mathrm{RE}=\mathrm{Y}, \mathrm{La}, \mathrm{Ce}, \mathrm{Pr}, \mathrm{Nd}$, Sm, Gd, Tb, Dy, Er or Yb), the Al-Sm binary alloy has been shown to exhibit GFA for the widest range of compositions (from 8at.\% to 16at.\% of Sm) [14]. For this reason, many experimental studies have been performed on Al-Sm binary alloys prepared by rapid quenching [15, 16, 17, 18, 19, 20]. However, understanding the SRO structure and its evolution during solidification as well as understanding the effect of the Sm concentration on the GFA of Al-Sm binary MGs are still open questions. These questions are addressed by performing molecular dynamics (MD) simulations of Al-Sm MGs with a range of stoichiometries and by characterizing their atomic structure during the solidification process.

Simulations are performed using the Finnis-Sinclair empirical potential implemented for the Al-Sm MGs [21] in the LAMMPS software package 22]. Lattice parameters and formation energies of $\mathrm{Al}$ rich crystalline compounds have been included in the development of this potential. Although these parameters have been fitted only for Sm concentrations of up to 25 at.\%, it will be shown that quantities, such as diffusion coefficient (DC), agree well with more accurate density functional theory (DFT) calculations for a wider range of compositions (up 30 to $50 \mathrm{at} . \% \mathrm{Sm})$. Therefore $\mathrm{Al}_{\mathrm{x}} \mathrm{Sm}_{100-\mathrm{x}}$ alloys were analyzed with compositions in the range of $100 \leq x \leq 50$. The Finnis-Sinclair potential has the following functional form $U=\sum_{i=1}^{N-1} \sum_{j=i+1}^{N} \theta_{t_{i} t_{j}}\left(r_{i j}\right)+\sum_{i=1}^{N} \Phi_{t_{i}}\left(\rho_{i}\right)$ where $t_{i}$ represents species of atom i, $\mathrm{N}$ is the total number of atoms, $\mathrm{r}_{\mathrm{ij}}$ is the distance between atoms $\mathrm{i}$ and $\mathrm{j}$ and $\theta_{\mathrm{t}_{\mathrm{i}} \mathrm{t}_{\mathrm{j}}}\left(\mathrm{r}_{\mathrm{ij}}\right)$ is the pairwise interaction energy. In the above equation, $\Phi_{\mathrm{t}_{\mathrm{i}}}\left(\rho_{\mathrm{i}}\right)$ is the embedding energy function and $\rho_{\mathrm{i}}=\sum_{\mathrm{j}} \Psi_{\mathrm{t}_{\mathrm{i}} \mathrm{t}_{\mathrm{j}}}\left(\mathrm{r}_{\mathrm{ij}}\right)$ is the electronic density, which in turn can be written as the sum of the electronic density functions $\Psi_{\mathrm{t}_{\mathrm{i}} \mathrm{t}_{\mathrm{j}}}\left(\mathrm{r}_{\mathrm{ij}}\right)$ of the individual atoms. The starting system consists of 32,000 atoms arranged on a face-centered cubic (fcc) lattice with $20 \times 20 \times 20$ unit cells. 
Periodic boundary conditions are applied in all three directions. Initially, $\mathrm{Al}$ and $\mathrm{Sm}$ atoms are randomly distributed on the fcc lattice so that the ratio of $\mathrm{Al}$ to $\mathrm{Sm}$ atoms is consistent with the selected alloy composition and then the system is equilibrated at $300 \mathrm{~K}$ for $0.2 \mathrm{~ns}$. The sample is subsequently melted and equilibrated for $1 \mathrm{~ns}$ at $T=2,000 \mathrm{~K}$, several hundred degrees higher than the melting temperature. Simulations are performed in the isothermal-isobaric

${ }_{45}$ (NPT) ensemble where the temperature and pressure are controlled using the Nose-Hoover thermostat and barostat, respectively. Zero pressure is maintained throughout all simulations. Subsequently, each system was quenched to $2 \mathrm{~K}$ in steps of $50 \mathrm{~K}$ with three different cooling rates of $4 \frac{\mathrm{K}}{\mathrm{ps}}, 2 \frac{\mathrm{K}}{\mathrm{ps}}$, and $1 \frac{\mathrm{K}}{\mathrm{ps}}$. The properties of the systems have been calculated for all the cooling rates but most of the results (unless otherwise noted) will be presented for the cooling rate of $1 \frac{\mathrm{K}}{\mathrm{ps}}$. At every $50 \mathrm{~K}$ interval, NPT simulations are performed for 15ps in order to equilibrate the system. After this equilibration time, NPT simulations are continued at the same temperature for another $15 \mathrm{ps}$ in order to calculate average properties of the systems. The timestep in the simulations is $1 \mathrm{fs}$. In addition, the calculated glass transition temperature $\left(T_{\mathrm{g}}\right)$ and the melting temperature $\left(T_{\mathrm{m}}\right)$ in the simulations were found to vary almost linearly from $520 \mathrm{~K}$ to $1,125 \mathrm{~K}$ and $T_{\mathrm{m}}$ monotonically from $933 \mathrm{~K}$ to $1,400 \mathrm{~K}$, respectively, between 0 and $50 \mathrm{at} . \% \mathrm{Sm}$.

Smaller Al-Sm systems (256 atoms) were used for ab initio MD (AIMD) simulations based on DFT. These systems were prepared by classical MD simulations starting from an fcc lattice with $4 \times 4 \times 4$ unit cells and periodic boundary conditions. $\mathrm{Al}$ and $\mathrm{Sm}$ atoms were distributed randomly on the fcc lattice. After that the systems were melted at $T=2,000 \mathrm{~K}$ and then cooled down to $2 \mathrm{~K}$, using classical MD simulations and NPT ensemble. Subsequently the samples were annealed inside the supercooled region by first gradually increasing the temperature to $T=1.1 \times T_{\mathrm{g}}$ (below $T_{\mathrm{m}}$ ) and then annealing for $0.5 \mathrm{~ns}$. Samples prepared this way were then used as an input for AIMD simulations. DFT calculations were carried out using the Vienna Ab Initio Simulation Package (VASP) 23, 24. AIMD simulations were performed in a canonical ensemble (constant volume and constant temperature), where the temperature is con- 
trolled using the Nose-Hoover thermostat and the timestep for the AIMD simulations is $1.5 \mathrm{fs}$. The $\Gamma$ point was used to sample the Brillouin zone of the supercell. The Projector Augmented Wave method and the Generalized Gradient Approximation [25, 26, 27] were used to describe the interacting valence electrons [28, 29]. The purpose of these AIMD simulations is to calculate DCs

75 and to demonstrate consistency for the empirical potentials. The samples were initially equilibrated by AIMD at $T=1.1 \times T_{\mathrm{g}}$ for $55 \mathrm{ps}$ and then the DC was calculated from the mean square displacement during the subsequent 20ps of the simulations. Finally, the ground state energy of systems created by DFT at RT was calculated using the conjugate-gradient minimization method.

The formation of MG was verified by calculating evolution of the total radial distribution function (RDF) throughout the solidification process. An example of such evolution for $\mathrm{Al}_{90} \mathrm{Sm}_{10}$ is shown in Fig. 1. Specifically, the RDF is given for the liquid state at $2,000 \mathrm{~K}$, supercooled system at $800 \mathrm{~K}$, and the system after solidification at $300 \mathrm{~K}$. The RDF is calculated at every $T$ by averaging the RDFs from the last 15ps of the NPT simulations. The plot in Fig. 1 shows that the system at $2,000 \mathrm{~K}$ is in a liquid state because the first peak is broad and there are no peaks at distances larger than $4 \AA$. Upon quenching to $800 \mathrm{~K}$, the first nearest neighbor peak becomes higher and narrower, which suggests the initiation of some type of ordering. The second peak at this temperature around $5 \AA$ starts to split (see inset in Fig. 1). Such splitting is characteristic of MG structures that incorporate SRO and MRO [30, 31, 32]. The sharpening of the first peak and splitting of the second peak are even more pronounced in the solidified structure quenched to room temperature (RT), which confirms MG formation. The RDF was also examined for other cooling rates studied in this work and found to have qualitatively similar distributions.

In order to gain further understanding into the effect of Sm on the atomic structure of the MG during solidification, the evolution of the atomic structure was monitored for various Sm concentrations as each system cools down to $2 \mathrm{~K}$. Voronoi tessellation technique 33] is a useful method to extract information about the system structure, especially the SRO. In this technique, a Voronoi 


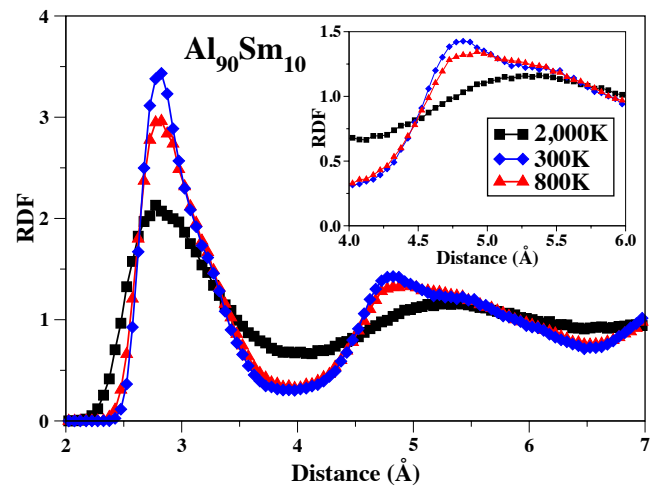

Figure 1: (Color online) Total radial distribution function (RDF) of $\mathrm{Al}_{90} \mathrm{Sm}_{10}$ for the liquid state at $2,000 \mathrm{~K}$, the supercooled region $800 \mathrm{~K}$, and the system after solidification at $300 \mathrm{~K}$. Inset: $\mathrm{RDF}$ of $\mathrm{Al}_{90} \mathrm{Sm}_{10}$ showing the splitting at the second peak.

polyhedral face is placed halfway between each pair of atoms. The edges of the polyhedron are defined by the intersection of these faces. The local structure can then be characterized by the number $\left(\mathrm{n}_{\mathrm{i}}\right)$ of the faces that contain a given number (i) of the edges in each VP. Each particle of the system can be viewed as the center of a VP and thus the indices can be assigned at every system atom. The VPs are grouped as: icosahedral-like (ICO-like), crystal-like, mixed and other VPs. If the number of polyhedral faces with 5 edges is larger or equal to $10\left(n_{5} \geq 10\right)$ then the VPs are classified as ICO-like. If this number is smaller than 5 and at the same time the numbers of polyhedral faces with 6 and 4 edges is each larger or equal to 3 , the VPs are labeled as crystal-like $\left(n_{4} \geq 3\right.$, $\left.n_{5} \leq 4, n_{6} \geq 3\right)$. Mixed VPs must have 3 polyhedral faces with 4 edges and 6 or more polyhedral faces with 5 edges $\left(n_{4}=3, n_{5} \geq 6\right)$. The remaining VPs are labeled as other VPs. The clusters exhibiting Al-like MRO are not treated in this analysis [13, 19]. In Fig. 2 the fractions of the ICO-like, crystal-like, mixed and other VPs are shown versus temperature for $\mathrm{Al}_{90} \mathrm{Sm}_{10}$ and $\mathrm{Al}_{50} \mathrm{Sm}_{50}$. For the low $\mathrm{Sm}$ concentration systems (i.e., $\mathrm{Al}_{90} \mathrm{Sm}_{10}$ ), the fraction of mixed clusters changes only from $34 \%$ to $36 \%$ from $1,500 \mathrm{~K}$ to $2 \mathrm{~K}$, and the fraction of the crystallike clusters decreases from $18.5 \%$ to $3.5 \%$ in the same temperature regime. At 
the same time, the fraction of other VPs also decreases from $43.4 \%$ to $17 \%$. On symmetry inside the supercooled liquid of the $\mathrm{Al}_{50} \mathrm{Sm}_{50}$ alloy is in contrast to the findings for the $\mathrm{Al}_{90} \mathrm{Sm}_{10}$ alloy. This result suggests that the glass formation is not promoted in the $\mathrm{Al}_{50} \mathrm{Sm}_{50}$ alloy. Finally, given that it was found exper- 


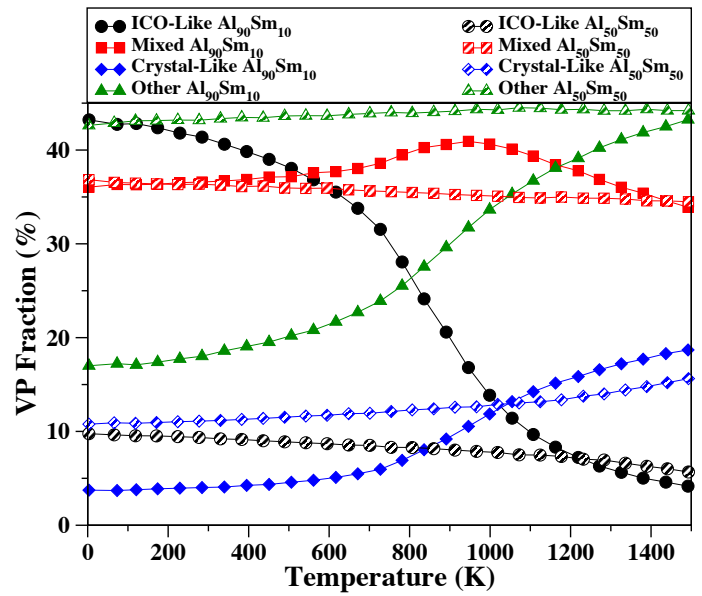

Figure 2: (Color online) Evolution of ICO-like, crystal-like, mixed, and other VPs during cooling down for $\mathrm{Al}_{90} \mathrm{Sm}_{10}$ and $\mathrm{Al}_{50} \mathrm{Sm}_{50}$ (solid and half solid symbols, respectively). It has been argued [6] that if there was a building unit (e.g., ICO-like cluster, 


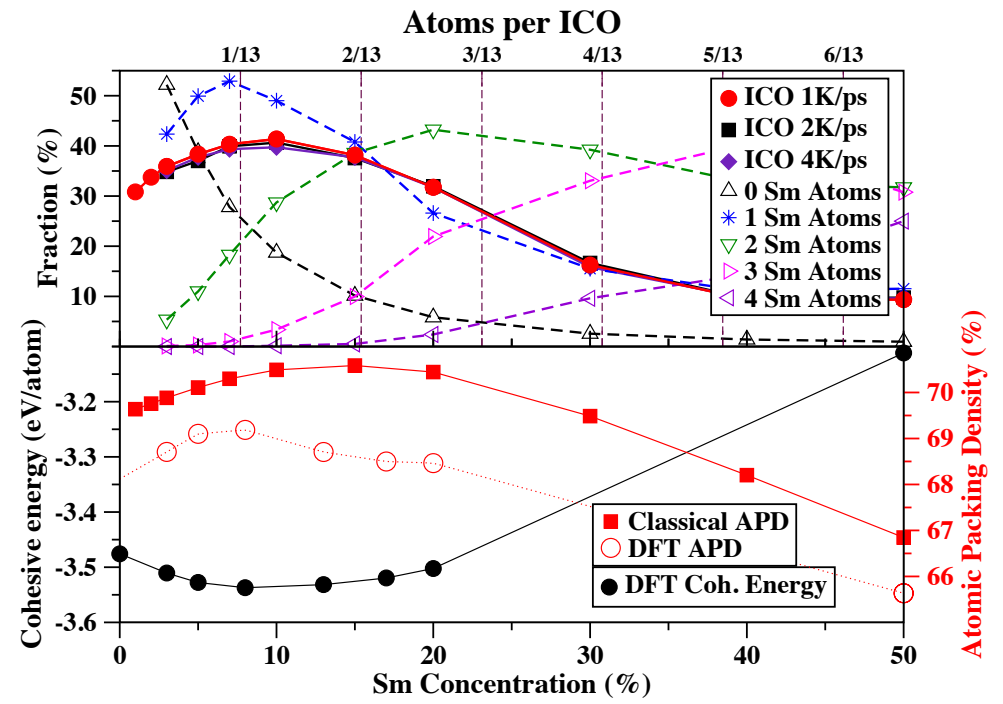

Figure 3: (Color online) (a) Solid symbols: fraction of ICO-like VPs for cooling rates $4 \frac{\mathrm{K}}{\mathrm{ps}}, 2 \frac{\mathrm{K}}{\mathrm{ps}}$ and $1 \frac{\mathrm{K}}{\mathrm{ps}}$, versus concentration of $\mathrm{Sm}$ (bottom horizontal axis). Open symbols correspond to the number of Sm atoms inside an ICO cluster, which consists of 13 atoms (top horizontal axis) at cooling rate $1 \frac{\mathrm{K}}{\mathrm{ps}}$. The top and bottom horizontal axes are not independent of each other as explained in the main text. (b) Cohesive energy calculation from DFT versus Sm concentration (black) and atomic packing density at $300 \mathrm{~K}$ versus Sm concentration calculated by MD and DFT (red). The systems were quenched down with the cooling rate $1 \frac{\mathrm{K}}{\mathrm{ps}}$. 
supercluster) of the MGs, its composition should be the same as the sample composition. This point is addressed by calculating the number of Sm atoms that exist inside the ICO-like clusters. In Fig. 3(a), the top horizontal-axis represents the number of Sm atoms inside an ICO-like cluster (each such cluster consists of 13 atoms), while the bottom horizontal axis represents concentration of Sm atoms inside each cluster. Dashed lines with open symbols correspond to the average number of $\mathrm{Sm}$ atoms that exist inside the ICO-like VPs for each composition of the sample. As the Sm concentration increases in the alloy, the number of Sm atoms inside the ICO-like clusters increases as well. There is only one $\mathrm{Al}-\mathrm{Sm}$ composition $\left(\mathrm{Al}_{93} \mathrm{Sm}_{7}\right)$, where the $\mathrm{Sm}$ concentration inside most of the ICO-like clusters (> 50at.\%, star symbols in Fig. 3(a)) matches the $\mathrm{Al}_{93} \mathrm{Sm}_{7}$ alloy composition. At this particular composition, there is also a peak in the fraction of $1 \mathrm{Sm}$ per ICO-like VP. The fact that the population of $\mathrm{Al}_{12} \mathrm{Sm}_{1}$ ICO-like clusters has a maximum for the $\mathrm{Al}_{93} \mathrm{Sm}_{7}$ system means that the compositions of the ICO-like VPs existing inside the $\mathrm{Al}_{93} \mathrm{Sm}_{7}$ alloy have the tendency to become the same as the system's total composition. This result might be another indicator of enhanced glass stability for this particular alloy composition.

It is interesting to ask what other properties may correlate with compositions that have good GFA. It has been proposed that atomic packing density (APD) is one of them. APD determines the packing state of the VPs as well as the structural stability [36] and it is considered to be an important factor that affects the MG's mechanical properties [36]. APD is defined as the fraction of volume that is occupied by particles APD $=\frac{\mathrm{n}_{\mathrm{Al}} * \mathrm{~V}_{\mathrm{Al}}+\mathrm{n}_{\mathrm{Sm}} * \mathrm{~V}_{\mathrm{Sm}}}{\text { Volume }}$ where $\mathrm{n}_{\mathrm{Al}}$ and $\mathrm{n}_{\mathrm{Sm}}$ represent the numbers of $\mathrm{Al}$ and Sm atoms, respectively, and $\mathrm{V}_{\mathrm{Al}}$ and $\mathrm{V}_{\mathrm{Sm}}$ are the atomic volumes of $\mathrm{Al}$ and $\mathrm{Sm}$ atoms. The APD of the systems at $300 \mathrm{~K}$ calculated by MD was found to have a broad maximum at $~ 15$ at.\% Sm as shown in Fig. 3(b) APD calculated by DFT after the conjugate-gradient relaxation exhibits a maximum at $\sim 8$ at.\% of Sm. One should point out that APD is different from the average density of the alloy, which we found to be a monotonically decreasing function of Sm concentration. Another property that 
has been suggested to correlate with GFA is the cohesive energy [37, which using DFT and as shown in Fig. 3(b) cohesive energy has a broad minimum at 10at.\% Sm.

In addition to structural properties of alloys, it has been proposed [4] that kinetic properties, such as the propensity for motion, of VPs might also affect the GFA of MGs. To test this hypothesis, the DCs of Al were calculated using both classical MD and AIMD calculations at $T=1.1 \times T_{\mathrm{g}}$, i.e., inside the supercooled liquid. The results are shown in Fig. 4(a) and they are compared with previous DCs calculated by AIMD at constant $T=850 \mathrm{~K}$ [38, 39]. DCs calculated by the two methods are in a good agreement in each other, which demonstrates that the empirical potential used here is reliable in reproducing kinetic properties of the Al-Sm alloys. The DCs of the Al atoms that lie at the center of ICO-like VPs are compared with the DCs of the remaining $\mathrm{Al}$ atoms in the sample. The central Al atoms have lower DCs than the DCs of the remaining $\mathrm{Al}$ atoms in the sample, especially for compositions below $15 \mathrm{at} . \% \mathrm{Sm}$ amorphous phase due to their slow kinetics inside the supercooled region, for a specific Al-Sm composition. These results are in good agreement with previous studies 4], which showed that the ICO clusters are responsible for the dynamical slowdown of the supercooled liquid. Finally, it is interesting to look at the trend shown in Fig. 4 there is a monotonic decrease of the diffusion coefficient with increasing Sm concentration. The monotonic change in diffusion coefficient is inconsistent with the experimental prediction that the best glass formers are in the range 8-16at.\% of $\mathrm{Sm}$ and therefore this result indicates that the DC cannot 225 be used as a glass former predictor across different alloy compositions.

In conclusion, during the solidification process, the Sm presence (until 10at.\%) was found to promote the icosahedral ordering in the supercooled region. At the same time the DCs of the central Al atoms of the ICO-like VPs is lower than the DCs of the remaining $\mathrm{Al}$ atoms in the sample. The increase of the ICO-like 


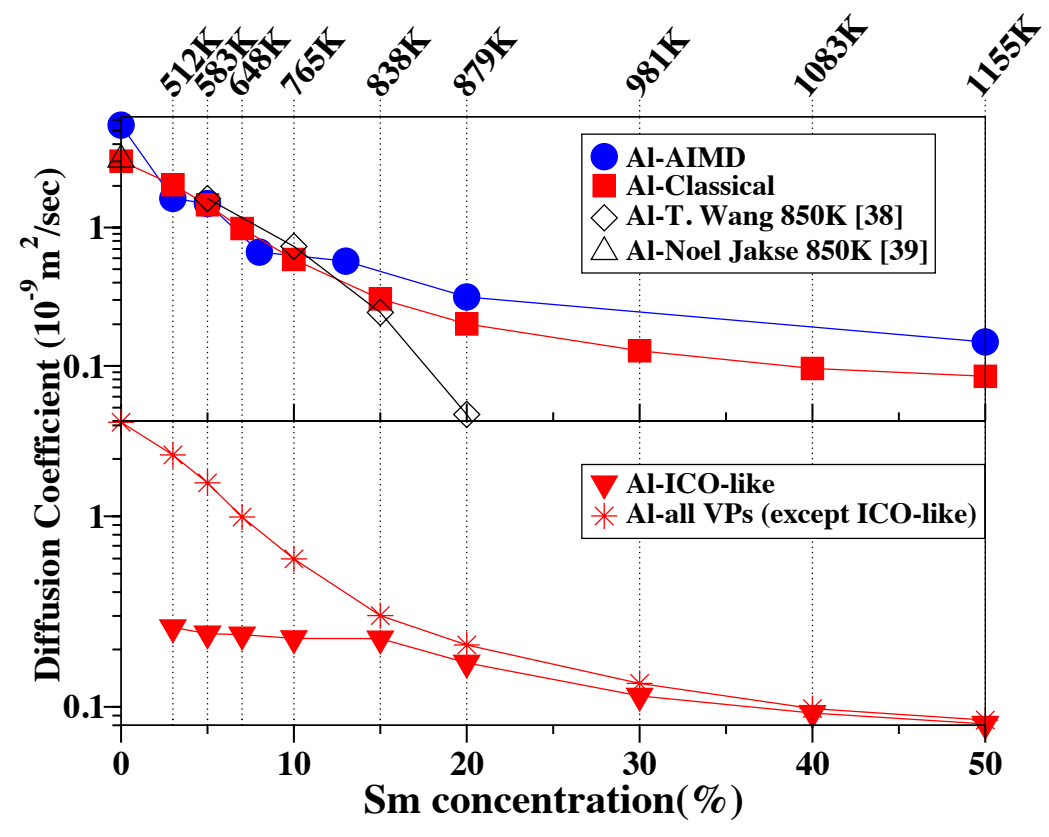

Figure 4: (Color online) (a) Diffusion coefficient calculated by classical MD and by ab initio $\mathrm{MD}$ for $\mathrm{Al}$ atoms versus Sm concentration. Top horizontal axis correspond to the temperature of the calculation, which is equal to 1.1 of Tg. Open symbols correspond to Al diffusion coefficient calculated from previous works at $850 \mathrm{~K}$. (b) Diffusion coefficient calculated by classical $\mathrm{MD}$ for the ICO-Like VP Al central atoms (triangles) and for the remaining $\mathrm{Al}$ atoms in the sample (star symbols). 

the ICO-like VPs in the alloys, results in slower relaxation dynamics inside the supercooled region. When ICO-like clusters are present, the alloys need more time to create crystal structure during solidification and thus these clusters can be correlated with better GFA and for the enhanced stability of the amorphous (above 10at.\%) to the alloy does not lead to an additional increase of icosahedral ordering because the DC is very low. After quenching the alloy to RT, the Sm concentration inside the Al-Sm MG was found to have a significant effect on the icosahedral ordering, the atomic packing density and the cohesive energy. Specifically, there is a region between 10 and 15at.\% Sm where the fraction of the ICO-like VPs and the atomic packing density are maximized, while the cohesive energy has a minimum in the same concentration regime. This outcome is consistent with experimental results that reported the glass formation range of the Al-Sm alloys as 8-16at.\% Sm. Finally, the presence of structural motifs 245 (i.e., of ICO-like VPs) correlates strongly with GFA, whereas kinetic properties (such as diffusion) are not strong predictors of GFA.

\section{Acknowledgments}

The authors acknowledge financial support from NSF-DMREF grant DMR1332851.

\section{References}

[1] A. Inoue, Acta Mater. 48 (2000) 279-306. doi 10.1016/S1359-6454(99) 00300-6

п[2] M. F. Ashby, A. L. Greer, Scr. Mater. 54 (2006) 321-326. doi $10.1016 / j$. scriptamat.2005.09.051.

[3] J. Perepezko, R. Hebert, Jom-Journal Miner. Met. Mater. Soc. 54 (2002) 34-39. 
[4] Y. Q. Cheng, E. Ma, H. W. Sheng, Appl. Phys. Lett. 93 (2008) 10-13. doi $10.1063 / 1.2987727$.

q [5] M. H. Cohen, D. Turnbull, Nature 189 (1961) 131-132. doi 10.1038/ 189131b0.

[6] G. A. Almyras, C. E. Lekka, N. Mattern, G. A. Evangelakis, Scr. Mater. 62 (2010) 33-36. doi $10.1016 /$ j.scriptamat.2009.09.019.

[7] G. B. Bokas, A. E. Lagogianni, G. A. Almyras, C. E. Lekka, D. G. Pan pageorgiou, G. A. Evangelakis, Intermetallics 43 (2013) 138-141. doi 10. $265 \quad 1016 / j$. intermet.2013.07.022.

[8] D. B. Miracle, Nat. Mater. 3 (2004) 697-702. doi:10.1038/nmat1219.

¿ [9] Y. Q. Cheng, E. Ma, H. W. Sheng, Phys. Rev. Lett. 102 (2009) 1-4. doi:10. 1103/PhysRevLett.102.245501.

[10] H. W. Sheng, W. K. Luo, F. M. Alamgir, J. M. Bai, E. Ma, Nature 439 (2006) 419-425. doi:10.1038/nature04421.

[11] C. E. Lekka, G. B. Bokas, G. A. Almyras, D. G. Papageorgiou, G. A.

口 Evangelakis, in: J. Alloys Compd., volume 536, pp. S65-S69. doi 10.1016/ j.jallcom.2011.11.038.

[12] C. Li, Y. Wei, X. Shi, Sci. Rep. 5 (2015) 12177. doi 10.1038/srep12177.

[13] P. Zhang, J. J. Maldonis, M. Besser, M. Kramer, P. M. Voyles, Acta Mater.

口 109 (2016) 103-114. URL: http://linkinghub.elsevier.com/retrieve/ pii/S1359645416300799 doi:10.1016/j.actamat.2016.02.006.

[14] A. Inoue, Prog. Mater. Sci. 43 (1998) 365-520. doi 10.1016/ S0079-6425(98)00005-X.

${ }_{280}$ [15] G. Wilde, H. Sieber, J. H. Perepezko, J. Non. Cryst. Solids 252 (1999) 621-625. 
[16] Y. E. Kalay, L. S. Chumbley, M. J. Kramer, I. E. Anderson, Intermetallics 18 (2010) 1676-1682. doi:10.1016/j.intermet.2010.05.005

[17] J. H. Perepezko, R. J. Hebert, R. I. Wu, G. Wilde, J. Non. Cryst. Solids 317 (2003) 52-61. doi:10.1016/S0022-3093(02)01983-X.

[18] J. H. Perepezko, W. Tong, J. Hamann, R. J. Hebert, H. R. Rosner, G. Wilde, Mat. Res. Soc. Symp. Proc. Vol. 754754 (2003) CC10.3.

[19] W. G. Stratton, J. Hamann, J. H. Perepezko, P. M. Voyles, X. Mao, S. V. Khare, Appl. Phys. Lett. 86 (2005) 141910. doi 10.1063/1.1897830.

[20] G. Wilde, H. Sieber, J. H. Perepezko, Scr. Mater. 40 (1999) 779-783. doi:10.1016/S1359-6462(99)00029-9.

[21] M. I. Mendelev, F. Zhang, Z. Ye, Y. Sun, M. C. Nguyen, S. R. Wilson, C. Z. Wang, K. M. Ho, Model. Simul. Mater. Sci. Eng. 23 (2015) 045013. doi $10.1088 / 0965-0393 / 23 / 4 / 045013$.

[22] S. Plimpton, J. Comput. Phys. 117 (1995) 1-19. doi:10.1006/jcph.1995. 1039 .

[23] G. Kresse, J. Furthmüller, Phys. Rev. B 54 (1996) 11169-11186. doi 10. 1103/PhysRevB.54.11169.

[24] G. Kresse, Phys. Rev. B 59 (1999) 1758-1775. doi:10.1103/PhysRevB.59. 1758 .

[25] J. Perdew, J. Chevary, S. Vosko, K. Jackson, M. Pederson, D. Singh,

口 C. Fiolhais, Phys. Rev. B 48 (1993) 4978-4978. doi 10.1103/PhysRevB. 48.4978 .2 .

[26] A. D. Becke, Phys. Rev. A 38 (1988) 3098-3100. doi:10.1103/PhysRevA. 38.3098

[27] D. C. Langreth, M. J. Mehl, Phys. Rev. B 28 (1983) 1809-1834. doi:10. 1103/PhysRevB.28.1809. 
[28] A. K. Rajagopal, J. Callaway, Phys. Rev. B 7 (1973) 1912-1919. doi:10. 1103/PhysRevB.7.1912.

[32] A. E. Lagogianni, G. Almyras, C. E. Lekka, D. G. Papageorgiou,

[33] J. L. Finney, Proc. R. Soc. London A Math. Phys. Eng. Sci. 319 (1970)

[34] Y.-F. Mo, R.-S. Liu, Z.-A. Tian, Y.-C. Liang, H.-T. Zhang, Z.-Y. Hou, H.R. Liu, A.-l. Zhang, L.-L. Zhou, P. Peng, Z. Xie, Phys. B Condens. Matter (2015) 1-8. doi:10.1016/j.physb.2015.02.020.

[35] G. Duan, D. Xu, Q. Zhang, G. Zhang, T. Cagin, W. L. Johnson, W. A.

[36] K. W. Park, J. I. Jang, M. Wakeda, Y. Shibutani, J. C. Lee, Scr. Mater. 57 (2007) 805-808. doi $10.1016 /$ j.scriptamat.2007.07.019

[37] K. J. Laws, K. F. Shamlaye, K. Wong, B. Gun, M. Ferry, Metall. Mater. 330

[38] N. Jakse, A. Pasturel, Sci. Rep. 3 (2013) 3135. doi 10.1038/srep03135. 
[39] T. Wang, F. Zhang, L. Yang, X. W. Fang, S. H. Zhou, M. J. Kramer, C. Z.

n Wang, K. M. Ho, R. E. Napolitano, Sci. Rep. 5 (2015) 10956. doi 10.1038/ 


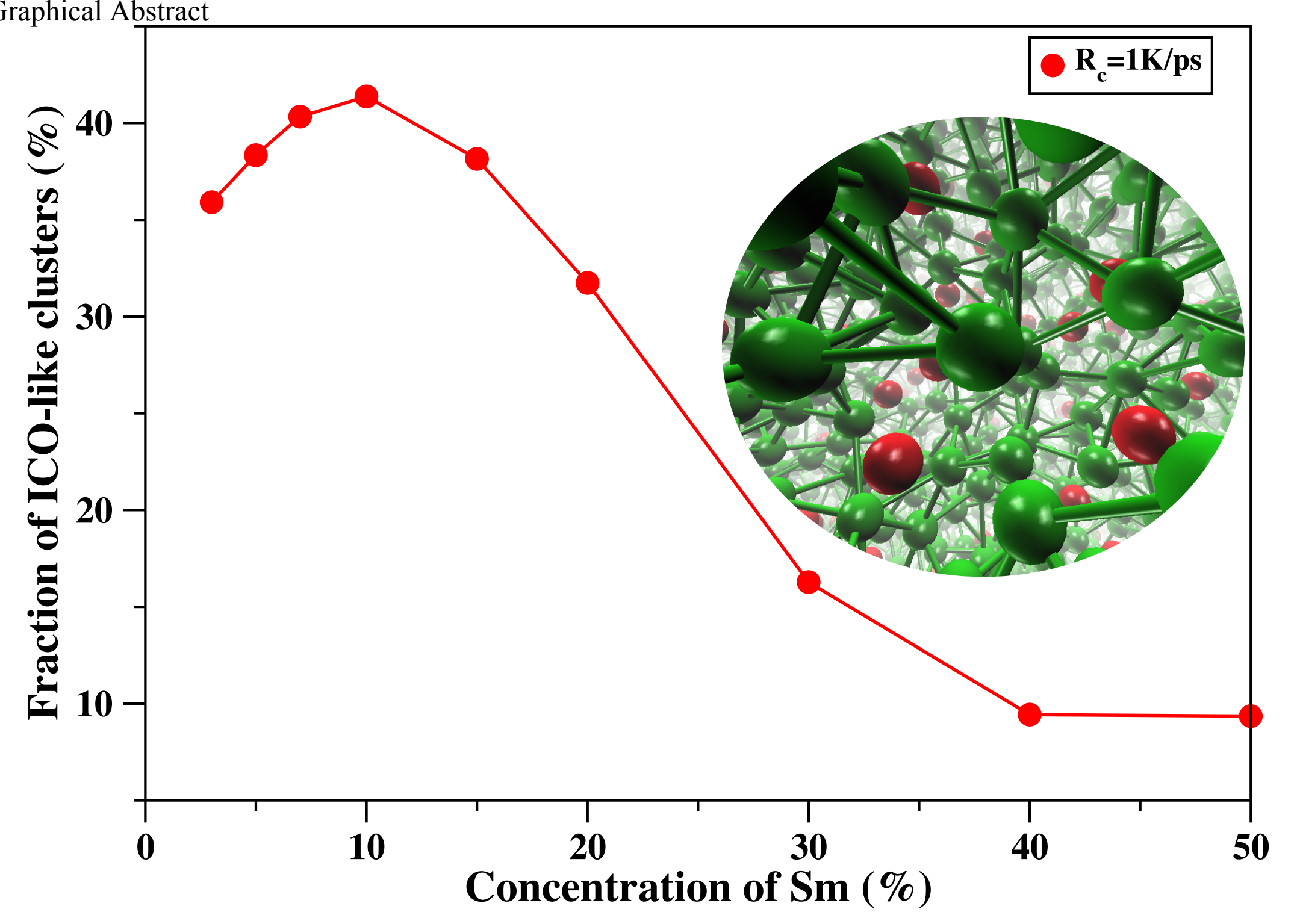

